\title{
Effectiveness of same-day antiretroviral therapy initiation in retention outcomes among people living with human immunodeficiency virus in Ethiopia: empirical evidence
}

\author{
Ismael Ahmed ${ }^{1 *}$ D, Meaza Demissie ${ }^{2}$, Alemayehu Worku ${ }^{3}$, Salem Gugsa ${ }^{4}$ and Yemane Berhane ${ }^{2}$
}

\begin{abstract}
Background: In August 2016, Ethiopia endorsed a universal "test and treat" strategy for people living with human immunodeficiency virus (PLHIV) based on World Health Organization recommendation. However, there is limited evidence on the routine application of the same-day "test and treat" recommendation in low-income settings. This study assessed the effect of same-day treatment initiation on individual-level retention at 6- and 12-months follow-up.

Methods: A multicenter facility-based retrospective cohort study was conducted to compare retention-in-care between PLHIV who started antiretroviral therapy (ART) on the same-day and those started ART $>7$ days following HIV diagnoses. Participants were at least 15 years-old and were newly diagnosed and started on ART between October 2016 and July 2018 in 11 health facilities in the Amhara region of Ethiopia. Multivariable logistic regression controlling for potential confounders and Kaplan-Meier survival analysis were used to assess differences in outcomes between the groups.

Results: In total, 433 PLHIV started ART on the same-day of diagnosis and 555 PLHIV who started ART $>7$ days after HIV diagnosis were included in the study. At 6-months, $82.0 \%$ (355) in the same-day group vs $89.4 \%$ (496) in the $>7$ days group were retained-in-care (absolute risk difference $(\mathrm{RD})=7.4 \%$; $95 \%$ confidence interval $(\mathrm{Cl})$ : $2.9-11.8 \%)$. At 12months, $75.8 \%$ (328) in the same-day group vs $82.0 \%$ (455) in the $>7$ days group were retained-in-care (absolute RD $=$ $6.2 \% ; 95 \% \mathrm{Cl}: 1.1,11.4 \%)$. The major drop in retention was in the first 30 days following ART initiation among same-day group. After adjusting for baseline and non-baseline covariates, the same-day group was less likely to be retained-incare at 6 - and 12-months (adjusted risk ratio $(\mathrm{RR})=0.89 ; 95 \% \mathrm{Cl}: 0.87,0.90$ and adjusted RR $=0.86 ; 95 \% \mathrm{Cl}: 0.83,0.89$, respectively).

Conclusions: Reduced retention-in-care can threaten the benefit of the same-day "test and treat" policy. The policy needs to be implemented cautiously with greater emphasis on assessment and preparation of PLHIV for ART to ensure treatment readiness before starting them on same-day ART and close monitoring of patients during early follow-up periods.
\end{abstract}

Keywords: Same-day antiretroviral therapy, Rapid ART, Retention, Attrition, Test and treat, Africa, Ethiopia

\footnotetext{
*Correspondence: ismaelahmed2003@gmail.com

'University of Gondar, Gondar, Ethiopia

Full list of author information is available at the end of the article
}

(C) The Author(s). 2020 Open Access This article is licensed under a Creative Commons Attribution 4.0 International License, which permits use, sharing, adaptation, distribution and reproduction in any medium or format, as long as you give appropriate credit to the original author(s) and the source, provide a link to the Creative Commons licence, and indicate if changes were made. The images or other third party material in this article are included in the article's Creative Commons licence, unless indicated otherwise in a credit line to the material. If material is not included in the article's Creative Commons licence and your intended use is not permitted by statutory regulation or exceeds the permitted use, you will need to obtain permission directly from the copyright holder. To view a copy of this licence, visit http://creativecommons.org/licenses/by/4.0/ The Creative Commons Public Domain Dedication waiver (http://creativecommons.org/publicdomain/zero/1.0/) applies to the data made available in this article, unless otherwise stated in a credit line to the data. 


\section{Background}

Despite increased access to and utilization of human immunodeficiency virus (HIV) testing and treatment services, attrition after being initiated on antiretroviral therapy (ART) has been a prevailing challenge for ART programs in low-income settings (LIS). Various systematic reviews conducted on ART programs in low- and middleincome countries $[1,2]$ and specifically in Ethiopia $[3,4]$ show high attrition from ART programs due to death or loss to follow-up (LTFU). Patients started on ART with advanced World Health Organization (WHO) clinical stage (III/IV) or bed ridden/seriously ill functional status [5-8] or lower CD4 cell count [8-10] had increased risk of attrition. This was mainly linked with delayed treatment initiation [5-7] that resulted from restricted initiation criteria following the earlier WHO guidance [11]. Increased mortality was also observed during the first 6-months of ART initiation $[5-7,9]$ which is likely related to the late presentation of patients for ART initiation [9].

Since 2013, evidence have emerged showing that early initiation of ART results in better clinical outcomes for people living with HIV (PLHIV) compared to delayed treatment in LIS [12-14]. Accordingly, in 2016, the WHO released a recommendation to provide universal "test and treat" services for PLHIV, regardless of their CD4 cell count or WHO clinical stage, in order to improve clinical outcomes. In addition, this universal eligibility strategy aimed to increase access to antiretroviral (ARV) drugs for treating and preventing HIV, and thereby achieve the "goal of ending the HIV epidemic as a major public health threat by 2030" [15].

Ethiopia adopted "immediate" ART initiation in August 2016 [16], and revised the national consolidated ART guideline again in August 2018 with more clear guidance on how soon to start treatment, including same-day ART initiation [17] following a WHO update that supports rapid ART initiation (within 1 week of HIV diagnosis) in July 2017 [18]. During this transitional time, some clinicians and HIV program managers were reluctant to initiate individuals on ART on the same-day of HIV diagnosis due to fears of poor adherence and attrition.

As same-day "test and treat" is a new initiative in LIS, there is a dearth of evidence regarding the effectiveness of ART initiation on the day of HIV diagnosis. Findings from the available Randomized Controlled Trails (RCTs) conducted on same-day ART initiation in Haiti [19] and Lesotho [20] are hard to extrapolate to ART clinics that lack basic resources for routine service delivery. Recent observational studies that evaluated the national scaleup of "test and treat" in South Africa [21] and Haiti [22] highlighted that although a higher proportion of PLHIV were started on ART on the same-day of diagnosis, those started on ART on same-day of HIV diagnosis were less likely to be retained-in-care 6-months post- initiation of treatment in the initial year of implementing "test and treat". A similar program evaluation in Nigeria reported that one-third of PLHIV who initiated on ART within 2 weeks of HIV diagnosis were LTFU, of whom more than half were lost within the first 30-days [23]. On the other hand, emerging results from studies in Africa have shown that certain group of individuals such as PLHIV with younger age [21, 23, 24], higher WHO clinical stage, higher baseline CD4 cell count, and physically affected functional status [23] have increased risk of LTFU after rapid ART initiation. Overall, despite the success of increased proportions of PLHIV enrolling for ART on the same-day of diagnosis [21, 22, 25], retaining PLHIV on ART in routine program settings remains a challenge. This may be due to initiation of ART without adequate preparation of PLHIV and assessment of their fully informed readiness for lifelong treatment [17]. In this study we sought to describe 6- and 12-months retention-in-care in PLHIV who were initiated on ART on the same-day of HIV diagnosis vs $>7$ days after HIV diagnosis, and assess the differences in retention-in-care before and after adjustment for observed differences in the two groups.

\section{Methods \\ Study design}

We used a multicenter retrospective cohort study design to compare the clinical outcomes of individuals who started ART on the day of HIV diagnosis (exposed) versus initiation after 7 days (non-exposed). To do this, routine clinical service delivery data were abstracted from facility-based medical records.

\section{Study setting}

The study was conducted at public health facilities in Bahir Dar and Gondar, towns in the Amhara region of northwest Ethiopia. The region shares more than $30 \%$ of the national HIV burden [26]. Out of 16 ART service providing public facilities in the two towns, 11 health facilities (eight health centers and three hospitals) that had > 20 PLHIV on ART by the end of December 2018 were included in the study. We excluded one newly established health center that provided ART service mainly for transferred-in (TI) persons from other health facilities during the study period. The participating facilities provide comprehensive HIV services such as HIV prevention, testing, care and treatment and laboratory services. They also have access to centralized viral load testing laboratories in their respective towns. Following ART initiation, viral load testing is done to routinely monitor virologic suppression at 6-months, at 12-months and then every 12-months thereafter. HIV services are provided by multidisciplinary teams composed of physicians, health officers, nurses, pharmacy technicians, laboratory 
technicians, adherence case managers, adherence supporters, and data personnel. The ART initiation and followup services are mainly provided by nurses who underwent ART training based on the national consolidated ART training manual. According to the latest national ART guideline, PLHIV are immediately linked to an ART clinic for a confirmatory test, counseling, adherence preparation and rapid ART initiation - including same-day ART for persons who are ready to start ART at the first clinical visit and have no sign of opportunistic infections (OI) (e.g. Tuberculosis (TB) and cryptococcal meningitis) that would result in delayed ART initiation [17]. The guideline recommends baseline laboratory tests such as CD4 cell count, CBC, ALT, and creatinine, if available. PLHIV who have no sign of OI but deferred rapid ART initiation were counseled and given an appointment to return a week following their HIV diagnosis for initiation. This scheme - counseling and making an appointment for a person to start ART a week later - was similar to ART implementation prior to the "test and treat" era [27]. After initiation, PLHIV were asked to return to ART clinic for monitoring at $1 \frac{12-}{2}, 1-, 3-, 4-$, and 6-months, and every 3 months thereafter irrespective of time of ART initiation [17].

\section{Participants}

This study included two groups of PLHIV who were newly diagnosed and started on ART at the study sites; those who were started on the same-day as HIV diagnosis and those who were started $>7$ days after the initial diagnosis. Eligible PLHIV were $\geq 15$ years-old and newly initiated on ART between 20 October 2016 (the time when study sites started same-day ART initiation) and 18 July 2018. Records of ART clients who were initiated on ART 1-7 days after diagnosis, aged < 15 years old, pregnant, TI from another health facility, and transferredout (TO) to another health facility within 12-months of ART initiation were excluded from the study. In addition, persons whose rapid ART initiation was delayed for clinical reasons (e.g. TB and cryptococcal meningitis) [17] were excluded from the study.

\section{Variables and measurement}

The primary outcome of this study was retention-in-care at 6- and 12-months, which was defined as PLHIV known to be alive and receiving ART at the end of a follow-up periods $[17,28]$. LTFU and death at 6 - and 12 -months were secondary outcomes of the study. Individuals who did not refill their ART for a period of one or more month after their last refill appointment date and were not yet classified as having died or TO were labeled as LTFU. However, individuals who discontinued treatment but returned to care before 6- and 12-months post-ART initiation were considered as retained at 6and 12-months, respectively. Death was defined as all- cause death for clients whose death was recoded on the patient's medical record or ART register.

Independent factors considered in the analysis include biological, sociodemographic and clinical factors. Biological factors included CD4 cell count and body mass index (BMI). Sociodemographic factors included age, sex, marital status, education, religion, residence, partner's HIV status and disclosure of HIV status. WHO clinical stage, functional status, type of ARV regimen initiated and taking cotrimoxazole preventive therapy (CPT) and isoniazid preventive therapy (IPT) were used to measure clinical condition. All variables were measured at baseline except for: partner's HIV status which was measured by the 6-month time point and use of CPT and IPT by the 6- and 12-month time point. Type of ARV regimen was measured at ART initiation and if regimen was switched.

Data were abstracted from the patients' intake and follow-up forms completed during enrollment and routine follow-up in the ART clinics. The data were captured using the Open Data Kit electronic system to ensure efficient and validated data-entry, using preprogrammed options for a majority of the data entered [29]. Data of eligible persons were abstracted from medical records by 11 experienced ART prescriber nurses working at the ART clinics. Data quality assurance and on-site support for data abstraction was provided by the study team.

\section{Study size and sampling method}

The sample size was determined using StatCalc for cohort studies using Epi Info $^{\mathrm{m}}$ version 7 (developed by Centers for Disease Control and Prevention). We estimated the proportion of retention among PLHIV who started ART on the same-day of diagnosis and $>7$ days after diagnosis based on a study from South Africa [30]. Accordingly, $\mathrm{P}_{1}$ of $81 \%$ (proportion of retention at 10months of ART among persons in the same-day group), $\mathrm{P}_{2}$ of $64 \%$ (proportion of retention at 10-months of ART among individuals in the control group), a 1:1 proportion, $\alpha$ of 0.05 , and $80 \%$ power was used to calculate sample size. We used a design effect of 1.5 to account for the effect of clustering in this multicenter design and increased the required sample size by $15 \%$ allowance for anticipated limitations with regard to missing medical record data in Ethiopia [6]. Based on this, we estimated that we would need to enroll a minimum of 420 participants in both groups. However, in order to increase the power of the study, all eligible individuals who were newly diagnosed and started on ART between 20 October 2016 and 18 July 2018 were included in the study, making the total sample size 988 (433 same-day and 555 after 7 days) (Fig. 1). 


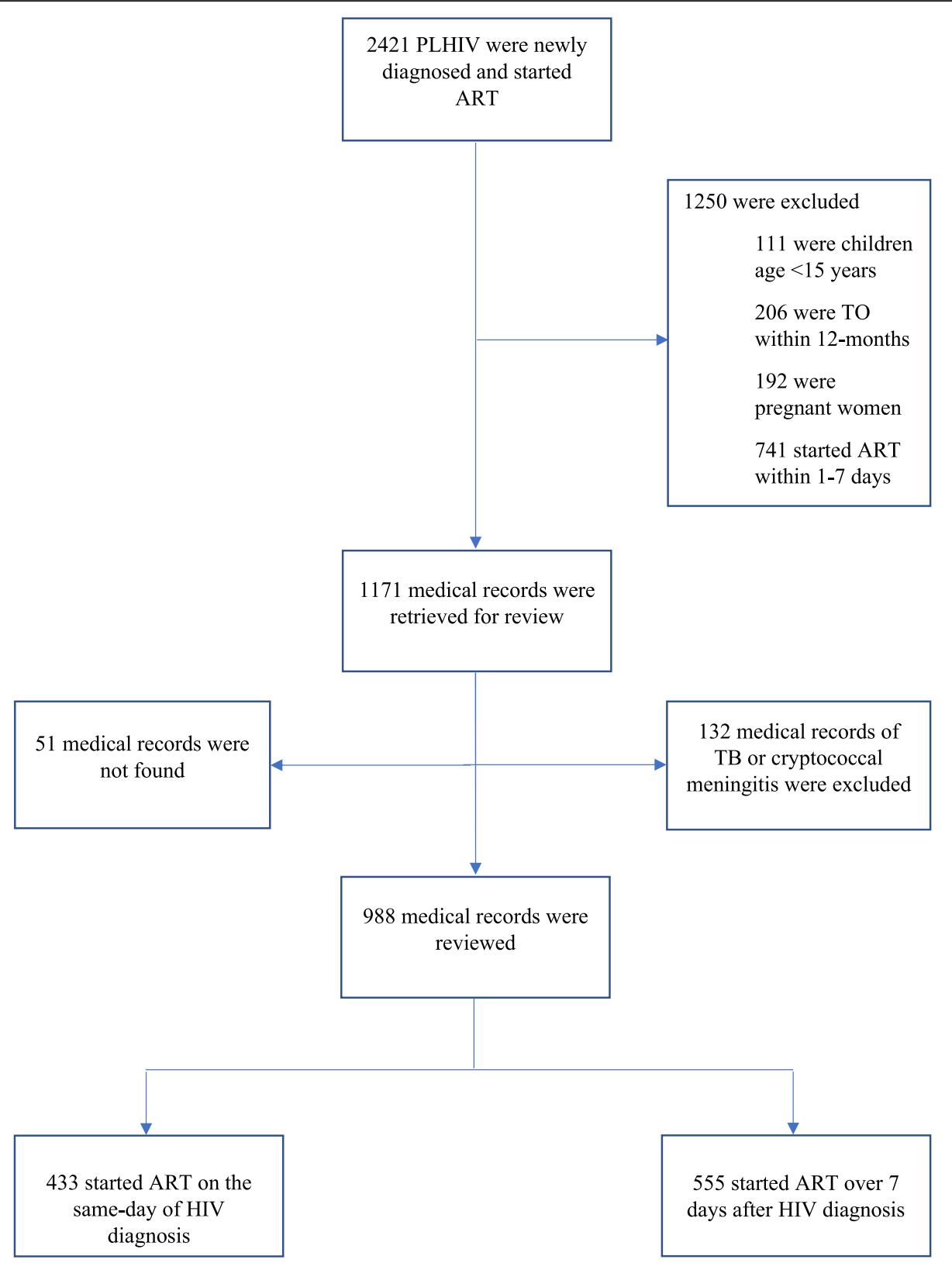

Fig. 1 Summary of number of participants and reasons for enrollment in the study

\section{Statistical analysis}

Data were exported to Stata version 13.0 (StataCorp., College Station, TX) for further cleaning and data analysis. Baseline characteristics of study participants were summarized using descriptive statistics. Proportions of persons retained-in-care, LTFU and died at 6- and 12months after enrollment were compared with absolute risk difference (RD) between the two groups using a chisquare test. Time-to-event methods (Kaplan-Meier plots and the log-rank test) were used to compare the cumulative probability of retention in comparison study groups. Person-time accrued from ART initiation date to the earliest of LTFU or death was estimated to compare incidence of attrition between the two groups.

We determined the missingness of the variables to be "missing completely at random" based on our knowledge of the HIV program and Little's chi-square test, and employed complete case analysis [31]. However, during analysis we excluded baseline CD4 cell count from the analysis due to a high proportion of missing data which was significantly different between the two comparison groups.

We compared the proportions of individuals achieving the primary (retention-in-care) and secondary (LTFU 
and death) outcomes at the end of 6- and 12-months using unadjusted and adjusted risk ratio (RR) with 95\% confidence interval (CI) for each study group.

We checked the balance between the two comparison groups using a chi-square test. To estimate the adjusted effects, we first balanced the two groups by estimating the propensity score using a logistic regression model $[32,33]$ to adjust for the baseline covariates such as age, sex, marital status, education, place of residence, BMI, WHO clinical stage, functional status and OI at enrollment. We used doubly-robust multivariable logistic regression model using backward stepwise variable selection that included both the propensity score and the baseline covariates to ensure sufficient covariate balance $[32,33]$. We also considered other non-baseline covariates in the model such as CPT, IPT, type of ARV regimen initiated, disclosure of HIV status and partner's HIV status. We accounted for the effect of clustering at town level using cluster-robust standard error [34, 35]. We explored all possible interaction terms between the main effect variables. The final model that included the propensity score and all significant variables $(p<0.05)$ was tested for goodness-of-fit using the HosmerLemeshow test supplemented by c-statistics for the area under the receiver operator characteristic curve [36]. Adjusted RR with 95\% CI was computed after running the final logit model [37].

We conducted a sensitivity analysis for the primary outcome by excluding individuals with WHO clinical stages III or IV to observe the effects of treatment in persons who were less likely to have advanced disease events [18]. The sensitivity analysis excluded 219 study participants (39 same-day and $180>7$ days starters) with WHO stage III or IV.

\section{Result}

\section{Characteristics of study participants}

Of the 2421 people who were newly diagnosed and started on ART at the participating health facilities between 20, October 2016 and 18, July 2018, a total of 433 same-day starters and 555 who started $>7$ days after the initial diagnosis were eligible to be included in our study (Fig. 1).

More than half $(52.6 \%, n=520)$ of study participants were women. The overall median age at ART initiation was 33 years (IQR 27, 40 years). The majority were married $(42.3 \%, n=396)$, resided in town $(80.8 \%, n=795)$, presented with WHO clinical stage I $(57.1 \%, n=564)$, had no OI at enrollment $(81.9 \%, n=809)$, and had a median BMI of 20 (IQR 18, 22). Of the total 988 study participants, only $57.8 \%(n=571)$ had documented baseline CD4 cell count result with significant missing data difference between the two groups (264 (61.0\%) in the same-day group and $153(27.6 \%)$ in the $>7$ days group; $p<.001)$.
Table 1 Sociodemographic characteristics of study participants by group (October 20, 2016 to July 18, 2018)

\begin{tabular}{|c|c|c|}
\hline Characteristics & $\begin{array}{l}\text { Same-day group } \\
\text { n (\%) }\end{array}$ & $\begin{array}{l}>7 \text { days group } \\
\mathrm{n}(\%)\end{array}$ \\
\hline \multicolumn{3}{|l|}{$\operatorname{Sex}(N=988)$} \\
\hline Male & $190(43.9)$ & $278(50.1)$ \\
\hline Female & $243(56.1)$ & $277(49.9)$ \\
\hline $\begin{array}{l}\text { Age in years }(N=988)- \\
\text { Median }(\mathrm{IQR})\end{array}$ & $32(27.0-39.0)$ & $34(28.0-40.0)$ \\
\hline \multicolumn{3}{|c|}{ Educational status $(N=924)^{\dagger}$} \\
\hline No Education & $107(26.4)$ & $154(29.7)$ \\
\hline Primary & $121(29.9)$ & $170(32.8)$ \\
\hline Secondary & $130(32.1)$ & $122(23.5)$ \\
\hline Tertiary & $47(11.6)$ & $73(14.1)$ \\
\hline \multicolumn{3}{|l|}{ Marital status $(N=936)$} \\
\hline Never married & $97(23.3)$ & $124(23.9)$ \\
\hline Married & $171(41.0)$ & $225(43.4)$ \\
\hline Divorced/separated & $126(30.2)$ & $147(28.3)$ \\
\hline Widow/er & $23(5.5)$ & $23(4.4)$ \\
\hline \multicolumn{3}{|l|}{ Religion $(N=979)$} \\
\hline Orthodox & $389(90.9)$ & $507(92.0)$ \\
\hline Protestant & $3(0.7)$ & $3(0.5)$ \\
\hline Muslim & $36(8.4)$ & $41(7.4)$ \\
\hline \multicolumn{3}{|c|}{ Place of residence $(N=984)^{\dagger}$} \\
\hline Within town & $368(85.2)$ & $427(77.4)$ \\
\hline Out of town & $64(14.8)$ & $125(22.6)$ \\
\hline \multicolumn{3}{|c|}{ Disclosure of HIV+ status $(N=728)^{*}$} \\
\hline Disclosed & $291(85.3)$ & $329(84.2)$ \\
\hline Not disclosed & $50(14.7)$ & $61(15.8)$ \\
\hline \multicolumn{3}{|c|}{ HIV status of partner $(N=862)^{*}$} \\
\hline HIV negative & $54(14.2)$ & $68(14.1)$ \\
\hline HIV positive & $99(26.0)$ & $114(23.7)$ \\
\hline Unknown & $46(12.1)$ & $58(12.1)$ \\
\hline No partner & $182(47.8)$ & $241(50.1)$ \\
\hline \multicolumn{3}{|c|}{ Functional status $(N=986)^{\dagger}$} \\
\hline Working & $418(96.5)$ & $479(86.6)$ \\
\hline Ambulatory & $14(3.2)$ & $52(9.4)$ \\
\hline Bed ridden & $1(0.2)$ & $22(4.0)$ \\
\hline
\end{tabular}

†Variables significantly different between the two groups at $P<0.05$ level, based upon $\mathrm{X} 2$ test for equality of proportions. *In subsequent follow-up visits post-treatment initiation

$H I V$ human immunodeficiency virus, IQR interquartile range

Baseline and follow-up socio-demographic and clinical characteristics of participants in each study group are shown in Tables 1 and 2. Persons who were initiated on ART on same-day of and $>7$ days after diagnosis were similar in sex, median age, marital status, religion, partner's HIV status, and disclosure. However, in the sameday group, there were higher proportions of individuals 
Table 2 Clinical characteristics of study participants by group (October 20, 2016 to July 18, 2018)

\begin{tabular}{|c|c|c|}
\hline Characteristics & $\begin{array}{l}\text { Same-day group } \\
\text { n (\%) }\end{array}$ & $\begin{array}{l}>7 \text { days group } \\
\mathrm{n}(\%)\end{array}$ \\
\hline BMI $(N=987)$-Median $(\mathrm{IQR})^{\dagger}$ & $20.1(18.3-22.3)$ & $19.6(17.5-22.0)$ \\
\hline \multicolumn{3}{|l|}{ CD4 cell count $/ \mathrm{mm}^{3}(N=571)^{\dagger}$} \\
\hline$<200$ & $58(34.3)$ & $218(54.2)$ \\
\hline $200-349$ & $31(18.3)$ & $86(21.4)$ \\
\hline$\geq 350$ & $80(47.3)$ & $98(24.4)$ \\
\hline \multicolumn{3}{|l|}{ WHO clinical stage $(N=988)^{\dagger}$} \\
\hline Stage I & $323(74.6)$ & $241(43.4)$ \\
\hline Stage ॥ & $71(16.4)$ & $134(24.1)$ \\
\hline Stage III & $35(8.1)$ & $141(25.4)$ \\
\hline Stage IV & $4(0.9)$ & $39(7.0)$ \\
\hline \multicolumn{3}{|l|}{ Ol at enrollment $(N=988)^{\dagger}$} \\
\hline Yes & $37(8.6)$ & $142(25.6)$ \\
\hline No & $396(91.5)$ & $413(74.4)$ \\
\hline \multicolumn{3}{|l|}{ CPT within 6-months $(N=988)^{\dagger} *$} \\
\hline Yes & $124(28.6)$ & $373(67.2)$ \\
\hline No & $30(6.9)$ & $17(3.1)$ \\
\hline Not eligible & $276(64.4)$ & $165(29.7)$ \\
\hline \multicolumn{3}{|c|}{ CPT within 12-months $(N=988)^{\dagger} * *$} \\
\hline Yes & $103(23.8)$ & $335(60.4)$ \\
\hline No & $43(9.9)$ & $44(7.9)$ \\
\hline Not eligible & $287(66.3)$ & $176(31.7)$ \\
\hline \multicolumn{3}{|l|}{ IPT within 6-months $(N=988)^{\dagger}$ * } \\
\hline Yes & $273(63.1)$ & $213(38.4)$ \\
\hline No & $142(32.8)$ & $275(49.6)$ \\
\hline Not eligible & $18(4.2)$ & $67(12.1)$ \\
\hline \multicolumn{3}{|c|}{ IPT within 12-months $(N=988)^{\dagger} * *$} \\
\hline Yes & $269(62.1)$ & $259(46.7)$ \\
\hline No & $139(32.1)$ & $229(41.3)$ \\
\hline Not eligible & $25(5.8)$ & $67(12.1)$ \\
\hline \multicolumn{3}{|l|}{ ARV regimen started $(N=988)^{\dagger}$} \\
\hline $\mathrm{TDF}+3 \mathrm{TC}+\mathrm{EFV}(\mathrm{FDC})$ & $431(99.5)$ & $533(96.0)$ \\
\hline$A Z T+3 T C+E F V$ & $1(0.2)$ & $10(1.8)$ \\
\hline Others & $1(0.2)$ & $12(2.2)$ \\
\hline
\end{tabular}

†Variables significantly different between the two groups at $P<0.05$ level, based upon $x 2$ test for equality of proportions. *Within 6-months of ART initiation. ${ }^{* *}$ Within 12-months of ART initiation

$A R V$ antiretroviral, $A Z T$ Zidovudine, $B M I$ body mass index, $C P T$ cotrimoxazole preventive treatment, FDC fixed dose combination, EFV Efavirenz, IPT isoniazid preventive therapy, IQR interquartile range, 3TC Lamivudine, OI opportunistic infection, TDF Tenofovir, WHO World Health Organization

with WHO clinical stage I $(74.6 \%(n=323)$ vs $43.4 \%$ $(n=241))$ and no OI $(91.5 \%(n=396)$ vs $74.4 \%(n=413))$ at enrollment compared to the $>7$ days group. In the $>$ 7 days group, higher proportions of study participants were taking CPT by 6 -months $(67.2 \%(n=373)$ vs $28.6 \%$ $(n=124))$ and 12 -months $(60.4 \%(n=335)$ vs $23.8 \%(n=$ 103)) time point compared to the same-day group. On the other hand, compared to PLHIV who started ART > 7 days of HIV diagnosis, there were higher proportions of individuals who were taking IPT within the first 6-months $(63.1 \%(n=273)$ vs $38.4 \% \quad(n=213))$ and 12 -months $(62.1 \% \quad(n=269)$ vs $46.7 \% \quad(n=259))$ of initiating same-day ART. While both groups of study participants started with a Non-nucleoside Reverse Transcriptase Inhibitors (NNRTI)-based regimens, almost all $(99.5 \%, n=431)$ of the same-day group started on a fixed-dose combination (FDC) of Tenofovir (TDF) + Lamivudine (3TC) + Efavirenz (EFV) compared to $96.0 \%(n=533)$ of the $>7$ days group. Due to side effects or drug toxicity, 11 people in the $>7$ days group had their regimen changed to a different first-line ART in the 12-months following ART initiation. In addition, one person's ART in each group was changed to second-line ART within 12months of ART initiation.

\section{Retention-in-care at 6- and 12-months of ART follow-up}

Retention at 6-months was $82.0 \%(n=355)$ in the sameday group and $89.4 \%(n=496)$ in the $>7$ days group, indicating an absolute RD of $7.4 \%$ (95\% CI: 2.9, $11.8 \%$; $p<.001)$. Similarly, 12-months retention was lower in the same-day group $(75.8 \%, n=328)$ compared to the $>$ 7 days group $(82.0 \%, n=455)$ with an absolute RD of 6.2\% (95\% CI: 1.1, 11.4\%; $p=.02$ ) (Table 3).

Figure 2 demonstrates the cumulative probability of retention in each study group over the 365 days following treatment initiation. It shows that the probability of retention was significantly lower in same-day group, $\left(P=.008\right.$, based on the log-rank test; corresponding $\mathrm{X}^{2}=$ $7.14)$. The major drop in retention probability was in the first 30-days among the same-day group. In other words, attrition was higher in the same-day group (8.8/personmonths) compared to the $>7$ days group (6.0/personmonths) with incidence rate ratio of 1.5 (95\% CI: 1.10, $2.0 ; p=.006)$ during the 12-months follow-up time.

Compared to the $>7$ days group, the unadjusted and adjusted RR of 6-months retention for same-day group were 0.92 (95\% CI: 0.87, 0.97; $p<0.001$ ) and 0.89 (95\% CI: 0.87, $0.90 ; p<.001)$, respectively. Similarly, the unadjusted RR of being retained at the end of 12-months of follow-up for the same-day group was 0.92 (95\% CI: $0.86,0.99 ; p=.02)$ compared to the $>7$ days group; the adjusted RR for this comparison was 0.86 (95\% CI: 0.83, 0.89; $p=<.00 l$ ) (Table 4).

\section{LTFU and mortality at the end of 6- and 12-months of ART follow-up}

As reported in Table 3, 6-months LTFU was significantly higher in the same-day group (14.5\%) compared to the $>7$ days group (5.9\%) with an absolute RD of $8.6 \%$ 
Table 3 Outcomes of ART initiation at 6- and 12-months ART follow-up by group

\begin{tabular}{|c|c|c|c|c|}
\hline \multirow[t]{2}{*}{ Outcomes } & \multicolumn{2}{|c|}{ No. (\%) of participants } & \multirow{2}{*}{$\begin{array}{l}\text { Absolute RD, } \\
\%(95 \% \mathrm{Cl})\end{array}$} & \multirow[t]{2}{*}{$p$-value } \\
\hline & Same-day $(n=433)$ & $>7$ days $(n=555)$ & & \\
\hline \multicolumn{5}{|l|}{ Primary outcomes } \\
\hline Retention at 6-months & $355(82.0)$ & $496(89.4)$ & $7.4(2.9,11.8)$ & $<0.001$ \\
\hline Retention at 12-months & $328(75.8)$ & $455(82.0)$ & $6.2(1.1,11.4)$ & 0.02 \\
\hline \multicolumn{5}{|l|}{ Secondary outcomes } \\
\hline LTFU at 6-months & $63(14.5)$ & $33(5.9)$ & $8.6(4.7,12.5)$ & $<0.001$ \\
\hline LTFU at 12-months & $87(20.1)$ & $62(11.2)$ & $8.9(4.3,13.5)$ & $<0.001$ \\
\hline Death at 6-months & $15(3.5)$ & $26(4.7)$ & $1.2(-1.2,3.7)$ & 0.34 \\
\hline Death at 12-months & $18(4.1)$ & $38(6.8)$ & $2.7(-0.1,5.5)$ & 0.07 \\
\hline
\end{tabular}

(95\% CI: 4.7, 12.5\%; $p<.001$ ). At 12-months, similarly LTFU was significantly higher in the same-day (20.1\%) compared to those initiated $>7$ days $(11.2 \%)$ with an absolute RD of $8.9 \%$ (95\% CI: 4.3, $13.5 \% ; p<.001)$. Furthermore, compared to $>7$ days group, the adjusted RR of 6 months LTFU for same-day group was 2.92 (95\% CI: $1.57,5.42 ; p=<.001)$. The adjusted RR of 12-months LTFU for the same-day group was 2.11 (95\% CI: 1.70, $2.61 ; p=<.001)$, compared to the $>7$ days group. There was no statistically significant differences or risk in mortality at 6- and 12-months between the two groups (Table 4).

\section{Sensitivity analysis}

In a sensitivity analysis that included only study participants with WHO clinical stage I and II, retention was significantly lower in the same-day group (Additional file 1). Retention at 6 -months was $82.2 \%(n=324)$ in same-day group and $92.3 \%(n=345)$ in $>7$ days group with an absolute RD of 9.8\% (95\% CI: 5.1, 14.4\%; $p<.001$ ). Similarly, at the end of 12-months follow-up time, retention was lower in the same-day group $(76.1 \%, n=300)$ compared to the $>7$ days group $(85.1 \%, n=319)$ with an absolute RD of 8.9\% (95\% CI: 3.4, 14.5\%; $p=.002$ ). The adjusted RR of 6months retention for the same-day group was 0.86 (95\% CI $0.81,0.90 ; p<.001)$ compared to the $>7$ days group. Similarly, the adjusted RR of 12-months retention for the same-day group was 0.86 (95\% CI: $0.83,0.89 ; p<.001)$ compared to the $>7$ days group.

\section{Discussion}

Our findings demonstrated that individuals who started ART on the same-day of HIV diagnosis had lower retention at 6-months $(82.0 \%$ vs $89.4 \%)$ and 12 -months (75.8\% vs $82.0 \%$ ) of ART follow-up compared to those who started ART > 7 days after their HIV diagnosis. The cumulative probability of retention was significantly lower in the same-day group whereby the major drop in retention happened in the first 30-days of same-day ART initiation. Overall, attrition in the same-day group (8.8/person-month) was greater than in those who initiated ART 1 week later (6.0/person-month).

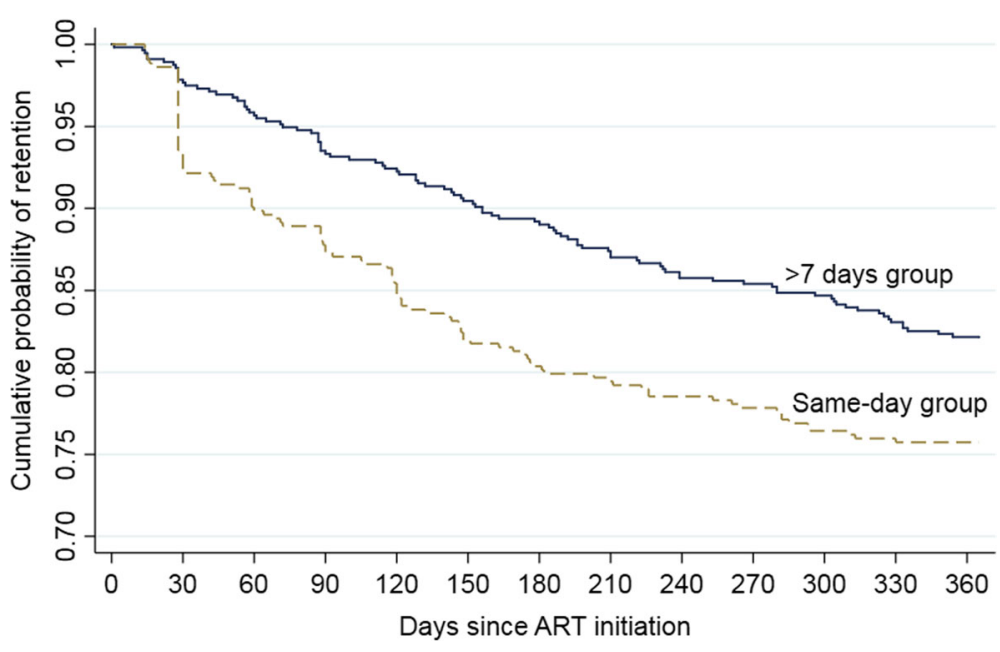

Fig. 2 Survival plots based on Kaplan-Meier estimates comparing retention since ART initiation by study groups 
Table 4 Unadjusted and adjusted RR of study outcomes for same-day ART group

\begin{tabular}{llllll}
\hline Outcomes & \multicolumn{2}{l}{ Unadjusted } & & \multicolumn{2}{l}{ Adjusted $^{* *}$} \\
\cline { 2 - 3 } & $\mathbf{R R}^{*}$ & $\mathbf{9 5 \%} \mathbf{C l}$ & & $\mathbf{R R}^{*}$ & $\mathbf{9 5 \%} \mathbf{C l}$ \\
\hline Retention at 6-months $^{\mathrm{a}}$ & 0.92 & $(0.87,0.97)$ & & 0.89 & $(0.87,0.90)$ \\
Retention at 12-months $^{\mathrm{b}}$ & 0.92 & $(0.86,0.99)$ & & 0.86 & $(0.83,0.89)$ \\
LTFU at 6-months $^{c}$ & 2.45 & $(1.64,3.66)$ & 2.92 & $(1.57,5.42)$ \\
LTFU at 12-months $^{\mathrm{d}}$ & 1.80 & $(1.33,2.43)$ & & 2.11 & $(1.70,2.61)$ \\
Mortality at 6-months $^{\mathrm{e}}$ & 0.74 & $(0.40,1.38)$ & & 1.26 & $(0.93,1.71)$ \\
Mortality at 12-months $^{\mathrm{f}}$ & 0.61 & $(0.35,1.05)$ & 0.94 & $(0.63,1.40)$ \\
\hline
\end{tabular}

LTFU Loss to follow-up, RR risk ratio

*Reference group = persons initiated on ART $>7$ days after HIV diagnosis ${ }^{*}$ Multivariable logistic regression model included the propensity score and other covariates such as:

aage, BMI, CPT, IPT, baseline OI, baseline functional status and partner's HIV status

${ }^{b}$ age, gender, BMI, IPT, baseline OI, baseline functional status, baseline WHO clinical stage, and partner's HIV status

'age, gender, education status, CPT, IPT, baseline OI and partner's HIV status

dage, gender, IPT, baseline OI, baseline WHO clinical stage and partner's HIV status

eage, educational status, BMI, baseline OI and baseline WHO clinical stage

fage, educational status, BMI, CPT, IPT and baseline functional status

The evidence in this study was generated using data from the routine clinical care settings in Ethiopia. We applied strict eligibility criteria to include study participants and involved multiple sites with a fairly large sample size [38]. We also used propensity score matching to balance the two comparison groups [33, 34]. So, we believe this study provides a fairly valid information on the subject matter under routine program conditions.

Even though two earlier RCTs demonstrated the efficacy of same-day ART initiation in improving retention of PLHIV at 12-months [19, 30], empirical observational studies raise concerns about whether the benefits measured in RCTs fully translate into real-world settings $[21,22]$. Our study, reflecting real-world clinical care settings, shows lower retention among individuals who start ART on the day of HIV diagnosis compared to those starting $>7$ days following diagnosis. Given the controlled and well-resourced nature of most RCTs, their generalizability to ordinary ART clinics is imperfect. For instance, in RCT conducted in Haiti [19] at a non-governmental urban clinic which was differently equipped in terms of infrastructure and staffing (physician evaluation and social worker counseling), participants were limited to PLHIV with disease in WHO clinical stages I or II and CD4 count $<500$ cells $/ \mathrm{mm}^{3}$ [19]. Additionally, participants were supported by accelerated counseling protocols, intensified early visit schedule, and transportation subsidy which are support systems that are rare during routine public health service provision [19]. In support of this argument, an observational study from the same setting (Haiti) found results that fell short of the benefit observed in the RCT [22].
Our study also demonstrated that there was significant attrition in the first month following same-day ART initiation. Similarly, more than half of LTFU PLHIV who initiated under "test and treat" strategy in Nigeria were LTFU within the first 30-days [23]. A high proportion of early program LTFU was also seen in South Africa where $35 \%$ of same-day initiators who were LTFU did not return to the ART program after the first initiation visit [21]. The Haiti observational study also showed that $20 \%$ of individuals who were initiated on same-day ART failed to return for ART refill after the initial ART dispensation [22]. These evidence show that there could be individuals who were not ready or not eligible for immediate ART initiation [15] but started on rapid ART without adequate assessment and support to address risk factors for attrition including barriers to treatment adherence $[39,40]$ and OI that may result in immune reconstitution inflammatory syndrome (IRIS) [41, 42]. In support of this argument, an RCTs of data from South Africa and Kenya showed that, when given an option, only half of newly diagnosed PLHIV were eligible and ready for same-day initiation [25]. Further studies are needed to investigate the reasons for the major drop in retention during the first 30-days following ART initiation.

Our study dichotomized attrition in terms of LTFU and death. Accordingly, we found that same-day ART initiators had close to 3 times higher risk of LTFU at 6months compared to $>7$ days group. Our findings were similar to "test and treat" program evaluation findings from South Africa, which reported increased odds of LTFU among same-day initiators compared with later initiators (started ART $\geq 1$ day) at the end of 6-months follow-up [21]. Additionally, we have observed statistically significant higher LTFU among same-day initiators compared to $>7$ days group at 12-months of follow-up. This is consistent with findings from a Nigerian study that showed a significant rate of LTFU at 12-months of ART initiation among PLHIV who started ART within 2 weeks [23]. These findings may point to the need for providing enhanced support and follow-up to PLHIV who started treatment on the initial date of HIV diagnosis. Our study showed no significant difference in the rate of mortality between the two groups at 6- and 12months periods. This finding was consistent with the systematic review conducted by Ford et al. among PLHIV who started on ART within 2 weeks of HIV diagnosis globally [12].

Despite a suboptimal retention rate and early program losses that have followed the scale-up of universal "test and treat" program especially same-day initiation, evidence showed an increased trend in implementation of rapid ART initiation in LIS [21, 22, 25]. While the push to meet the fast-tracked UNAIDS targets [43] has 
increased access to early treatment of PLHIV [44], the need for adequate preparation and readiness assessment of PLHIV is becoming evident. We echo the recommendation of Geng and Havlir [45] that the science of rapid ART should pay attention to the "how" part of treatment initiation since the effect of same-day treatment can be altered by patient, provider and system-level factors. More importantly, care should be taken not to force or unnecessarily encourage PLHIV to start treatment on the same-day of HIV diagnosis without ensuring patient comprehension of their result, the need for life-long commitment for treatment adherence and readiness for immediate ART initiation. PLHIV should be properly screened and treated for OI including advanced HIV disease before rapid ART initiation [18] to prevent possible death due to IRIS [41, 42]. Additionally, once initiated, provision of intensified client-centered support for adherence in the initial months of taking ART may be a key initiative to include given the high attrition in the first 30-days of taking ART. Furthermore, in the era of universal "test and treat", it is important to augment the routine facility-based clinical services with evidencebased initiatives that improve retention such as facilitybased adherence clubs [46, 47], community-based adherence support and extra community-based care for highrisk patients [46].

Our study has some limitations that need to be considered while interpreting the study findings. Firstly, given the retrospective nature of the study, we could not address important behavioral factors (e.g. substance use and mental health status) and other provider- and system-level independent factors that may affect the treatment outcomes. Secondly, routine clinical data are usually affected by incomplete information on some of the variables that could affect the analysis. For instance, we have excluded baseline CD4 result from the analysis due to differential missing data. However, this could be an important omission, since a study in Nigeria showed higher risk of LTFU among PLHIV who started ART with higher baseline CD4 cell count. In addition, a significant proportion of PLHIV who started ART under "test and treat" strategy had lower baseline CD4 cell count compared to previously enrolled PLHIV [23]. Since we could not adjust for the effect of this variable, our LTFU result may be overestimated. Thirdly, TO individuals who were referred to another facility within 12-months of ART initiation were excluded from the study due to difficulty to establish their follow-up status at $6^{\text {th }}$ and $12^{\text {th }}$ months. These people may have been less likely to continue their treatment in the other health facility [48]. Furthermore, TO cases were more common among PLHIV who started ART under "test and treat" strategy [23]. This may underestimate attrition due to potential additional loss among TO individuals [48].
Finally, caution should be taken while interpreting the LTFU and mortality results due the difficulty of ascertaining death from LTFU in Ethiopian clinical care settings [49] - some of individuals reported as LTFU could have been dead - leading to overestimation of LTFU and underestimation of death rates. This was also evidenced by a systematic review of studies that traced LTFU patients showing significant deaths among LTFU patients from sub-Saharan Africa ART program [50]. Particularly in Ethiopia, half of LTFU individuals traced through a community survey were found to be dead [49]. Despite these limitations, which are applicable to both groups compared, our study provides useful evidence on relationship between same-day ART initiation and retention outcomes in Ethiopia and builds the evidence-base on this research question in LIS.

\section{Conclusions}

In summary, we found significantly lower retention-incare among PLHIV who started ART on the same-day of HIV diagnosis at 6- and 12-months follow-up periods compared to those who started their treatment after 7 days. The major drop in retention was in the first 30days following ART initiation among same-day initiators compared to those who started treatment after 7 days. These findings imply the need for caution when initiating PLHIV on same-day ART while expanding access to treatment to end the public health threat of HIV epidemic by 2030 through rapid ART initiation. Reinforcing adequate preparation and assessment of individuals' readiness by healthcare workers before initiating PLHIV on same-day ART is vital to maximize retention among PLHIV starting treatment at the first clinical visit. Even with adequate assessment, the major lifestyle change in HIV diagnosis and initiation of a lifelong treatment may warrant enhanced client-centered adherence support and close monitoring of patients during early follow-up periods, particularly in the first 30-days of ART initiation.

\section{Supplementary Information}

The online version contains supplementary material available at https://doi. org/10.1186/s12889-020-09887-9.

Additional file 1: Table 1. Outcomes of ART initiation at 6- and 12months ART follow-up by group. Table 2. Unadjusted and adjusted RR of study outcomes for same-day ART group.

\section{Abbreviations}

APHI: Amhara Public Health Institute; ART: Antiretroviral Therapy; ARV: Antiretroviral; AZT: Zidovudine; FDC: Fixed Dose Combination; HIV: Human Immunodeficiency Virus; LIS: Low-income Setting; LTFU: Loss to Follow-up; NNRTI: Non-nucleoside Reverse Transcriptase Inhibitors; OI: Opportunistic Infections; PLHIV: People Living with HIV; RCTs: Randomized Controlled Trails; RD: Risk Difference; RR: Risk Ratio; 3TC: Lamivudine; TDF: Tenofovir; TI: Transferred-in; TO: Transferred-out; WHO: World Health Organization 


\section{Acknowledgements}

We would like to thank APHI, health facilities and data collectors for their support during the conduct of this study. Our deepest gratitude goes to Gizachew Tadesse for his special support on Open Data Kit electronic system and to Nancy Puttkammer for her critical review and comments on the manuscript. Finally, our special thanks go to Addis Continental Institute of Public Health and University of Gondar for giving the opportunity to study doctoral training program.

\section{Authors' contributions}

IA was the principal investigator of the study and had primary responsibility in conceptualization, protocol development, overseeing data collection, data cleaning and analysis, interpretation of results, and manuscript drafting and revision. MD had substantial contributions in the design of the study, protocol review, interpretation of results and critical review of the manuscript. AW had substantial contributions in the design of the study, protocol review, statistical analysis plan, synthesis of results and critical review of the manuscript. SG had substantial contributions in the review of study protocol, interpretation of results and critical review of the manuscript. YB had substantial contributions in the conceptualization and design of the study, protocol review, interpretation of results and critical review of the manuscript. All authors read and approved the manuscript.

\section{Funding}

The authors declare that they did not receive funding for this research from any source.

\section{Availability of data and materials}

The datasets used and/or analyzed during the current study are available from the corresponding author on reasonable request.

\section{Ethics approval and consent to participate}

Ethical clearance was obtained from the Institutional Review Board of the University of Gondar. The ethical clearance with the protocol was shared to Amhara Public Health Institute (APHI) for further clearance. APHI provided a support letter to town health offices requesting access to the medical records at study sites. PLHIV on ART were not directly contacted nor were their personal identifiers collected. Only data collectors and supervisors had access to the medical records and both groups signed confidentiality agreements before commencing data collection.

\section{Consent for publication}

Not applicable.

\section{Competing interests}

The authors declare that they have no competing interests.

\section{Author details}

${ }^{1}$ University of Gondar, Gondar, Ethiopia. ${ }^{2}$ Addis Continental Institute of Public Health, Addis Ababa, Ethiopia. ${ }^{3}$ Department of Preventive Medicine, School of Public Health, College of Health Sciences, Addis Ababa University, Addis Ababa, Ethiopia. ${ }^{4}$ Department of Global Health, University of Washington, Seattle, WA, USA.

Received: 31 July 2020 Accepted: 13 November 2020

Published online: 26 November 2020

\section{References}

1. Fox MP, Rosen S. Retention of Adult Patients on Antiretroviral Therapy in Low- And Middle-Income Countries: Systematic Review and Meta-analysis 2008-2013. J Acquir Immune Defic Syndr (1999). 2015; [cited 2020 Jun 2]. Available from: https://pubmed.ncbi.nlm.nih.gov/25942461/.

2. Fox MP, Rosen S. Patient retention in antiretroviral therapy programs up to three years on treatment in sub-Saharan Africa, 2007-2009: systematic review. Tropical Med Int Health. 2010;15:1-15.

3. Biset Ayalew M. Mortality and its predictors among HIV infected patients taking antiretroviral treatment in Ethiopia: a systematic review. AIDS Res Treat. 2017;2017:5415298.

4. Gesesew HA, Ward P, Hajito KW, Feyissa GT, Mohammadi L, Mwanri L. Discontinuation from antiretroviral therapy: a continuing challenge among adults in HIV care in Ethiopia: a systematic review and meta-analysis. PLoS One. 2017;12:e0169651 Public Library of Science.

5. Ayele W, Mulugeta A, Desta A, Rabito FA. Treatment outcomes and their determinants in HIV patients on anti-retroviral treatment program in selected health facilities of Kembata and Hadiya zones, southern nations, nationalities and peoples region, Ethiopia. BMC Public Health. 2015;15:826.

6. Melaku Z, Lamb MR, Wang C, Lulseged S, Gadisa T, Ahmed S, et al. Characteristics and outcomes of adult Ethiopian patients enrolled in HIV care and treatment: a multi-clinic observational study. BMC Public Health. 2015:15:462.

7. Mutasa-Apollo T, Shiraishi RW, Takarinda KC, Dzangare J, Mugurungi O, Murungu J, et al. Patient retention, clinical outcomes and attritionassociated factors of HIV-infected patients enrolled in Zimbabwe's National Antiretroviral Therapy Programme, 2007-2010. PLoS One. 2014;9:e86305.

8. Mekuria LA, Prins JM, Yalew AW, Sprangers MAG, Nieuwkerk PT. Retention in HIV care and predictors of attrition from care among HIV-infected adults receiving combination anti-retroviral therapy in Addis Ababa. PLoS One. 2015;10:e0130649.

9. Fekade D, Weldegebreal T, Teklu AM, Damen M, Abdella S, Baraki N, et al. Predictors of survival among adult Ethiopian patients in the national ART program at seven university teaching hospitals: a prospective cohort study. Ethiop J Health Sci. 2017;27:63-71.

10. Haile D, Takele A, Gashaw K, Demelash H, Nigatu D. Predictors of treatment failure among adult antiretroviral treatment (ART) clients in bale zone hospitals, South Eastern Ethiopia. PLoS One. 2016;11:e0164299.

11. WHO. Consolidated guidelines on the use of antiretroviral drugs for treating and preventing HIV infection [Internet].: WHO. World Health Organization; 2013. [cited 2020 Jul 21]. Available from: http://www.who.int/hiv/pub/ guidelines/arv2013/download/en/.

12. Ford N, Migone C, Calmy A, Kerschberger B, Kanters S, Nsanzimana S, et al. Benefits and risks of rapid initiation of antiretroviral therapy. AIDS (London, England). 2018; [cited 2020 Jun 2]. Available from: https://pubmed.ncbi.nlm. nih.gov/29112073/?from_single_result=Benefits+and+risks+of+rapid+ initiation+of+antiretroviral+therapy.

13. Brown LB, Havlir DV, Ayieko J, Mwangwa F, Owaraganise A, Kwarisiima D, et al. High levels of retention in care with streamlined care and universal test and treat in East Africa. AIDS. 2016;30:2855-64.

14. The INSIGHT START. Study group. Initiation of antiretroviral therapy in early asymptomatic HIV infection. New England journal of medicine. Mass Med Soc. 2015;373:795-807.

15. World Health Organization. Consolidated guidelines on the use of antiretroviral drugs for treating and preventing HIV infection: recommendations for a public health approach [Internet]. 2016 [cited 2020 May 5]. Available from: https://apps.who.int/iris/handle/10665/208825.

16. Federal Ministry of Health of Ethiopia. Supplement to the 2014 national comprehensive HIV prevention, care and treatment guideline of Ethiopia to address HIV test and start. 2016.

17. Federal Ministry of Health of Ethiopia. National consolidated guidelines for comprehensive HIV prevention, care and treatment [Internet]: WHO | Regional Office for Africa; 2018. [cited 2020 Jun 2]. Available from: https:// www.afro.who.int/publications/national-consolidated-guidelinescomprehensive-hiv-prevention-care-and-treatment.

18. World Health Organization. Guidelines for managing advanced HIV disease and rapid initiation of antiretroviral therapy [Internet]: WHO. World Health Organization; 2017. [cited 2020 Jun 2]. Available from: http://www.who.int/ hiv/pub/guidelines/advanced-HIV-disease/en/.

19. Koenig SP, Dorvil N, Dévieux JG, Hedt-Gauthier BL, Riviere C, Faustin M, et al. Same-day HIV testing with initiation of antiretroviral therapy versus standard care for persons living with HIV: a randomized unblinded trial. PLoS Med. 2017;14:e1002357.

20. Labhardt ND, Ringera I, Lejone TI, Klimkait T, Muhairwe J, Amstutz A, et al. Effect of offering same-day ART vs usual health facility referral during homebased HIV testing on linkage to care and viral suppression among adults with HIV in Lesotho: the CASCADE randomized clinical trial. JAMA. 2018;319: 1103-12.

21. Lilian RR, Rees K, McIntyre JA, Struthers HE, Peters RPH. Same-day antiretroviral therapy initiation for HIV-infected adults in South Africa: Analysis of routine data. PLoS One. 2020;15:e0227572 Public Library of Science.

22. Puttkammer N, Parrish C, Desir Y, Hyppolite N, Wagenaar B, Joseph N, et al. Toward universal HIV treatment in Haiti: time trends in ART retention after 
expanded ART eligibility in a National Cohort from 2011 to 2017. J Acquir Immune Defic Syndr. 2020;84:153-61.

23. Stafford KA, Odafe SF, Lo J, Ibrahim R, Ehoche A, Niyang M, et al. Evaluation of the clinical outcomes of the test and treat strategy to implement treat all in Nigeria: Results from the Nigeria multi-center ART study. PLoS One. 2019; 14:e0218555 Public Library of Science.

24. Brown LB, Getahun M, Ayieko J, Kwarisiima D, Owaraganise A, Atukunda $M$, et al. Factors predictive of successful retention in care among HIVinfected men in a universal test-and-treat setting in Uganda and Kenya: A mixed methods analysis. PLoS One. 2019;14:e0210126 Public Library of Science.

25. Rosen S, Maskew M, Larson B, Brennan A, Tsikhutsu I, Fox M, et al. Simplified Clinical Algorithm for Identifying Patients Eligible for Same-Day HIV Treatment Initiation (SLATE): Results From an Individually Randomized Trial in South Africa and Kenya. PLoS Med. 2019; [cited 2020 Jun 2]. Available from: https://pubmed.ncbi.nlm.nih.gov/31525187/.

26. Ethiopian Public Health Institute. HIV related estimates and projections for Ethiopia. 2018

27. Federal Ministry of Health of Ethiopia. National guidelines for comprehensive HIV prevention, care and treatment [Internet]. 2014. Available from: https://www.medbox.org/pdf/5e148832db60a2044c2d410d.

28. Rosen S, Fox MP, Gill CJ. Patient Retention in antiretroviral therapy programs in Sub-Saharan Africa: A systematic review. PLoS Med. 2007;4 [cited 2020 Sep 13]. Available from: https://www.ncbi.nlm.nih.gov/pmc/articles/PMC202 0494/.

29. Anokwa Y, Hartung C, Brunette W, Borriello G, Lerer A. Open source data collection in the developing world. Computer. 2009;42:97-9.

30. Rosen S, Maskew M, Fox M, Nyoni C, Mongwenyana C, Malete G, et al. Initiating antiretroviral therapy for HIV at a patient's first clinic visit: The RapIT randomized controlled trial. PLoS Med. 2016; [cited 2020 Jun 3]. Available from: https://pubmed.ncbi.nlm.nih.gov/27163694/.

31. Li C. Little's test of missing completely at random. Stat J. 2013;13:795-809 SAGE Publications.

32. Elze MC, Gregson J, Baber U, Williamson E, Sartori S, Mehran R, et al. Comparison of propensity score methods and covariate adjustment: evaluation in 4 cardiovascular studies. J Am Coll Cardiol. 2017:69:345-57.

33. Golinelli D, Ridgeway G, Rhoades H, Tucker J, Wenzel S. Bias and variance trade-offs when combining propensity score weighting and regression: with an application to HIV status and homeless men. Health Serv Outcome Res Methodol. 2012;12:104-18.

34. Mackinnon JG, Webb MD. When and how to deal with clustered errors in regression models [Internet]: Department of Economics Queen's University; 2019. [cited 2020 Aug 27]. Available from: http://qed.econ.queensu.ca/pub/ faculty/mackinnon/working-papers/qed_wp_1421.pdf.

35. Jayatillake RV, Sooriyarachchi MR, Senarathna DLP. Adjusting for a cluster effect in the logistic regression model: an illustration of theory and its application. J Natl Sci Foundation Sri Lanka. 2011;39:201-18.

36. Hosmer D, Lemeshow S. Applied logistic regression [Internet]. 2nd ed. United States of America: A Wiley-Interscience Publication; 2000. [cited 2020 Jun 3]. Available from: http://resource.heartonline.cn/20150528/1_3 kOQSTg.pdf.

37. Norton EC, Miller MM, Kleinman LC. Computing adjusted risk ratios and risk differences in Stata. Stat J. 2013:13:492-509 SAGE Publications.

38. Parfrey P, Barrett B. Clinical epidemiology: practice and methods. Second. New York: Springer Science+Business Media; 2015.

39. Bezabhe WM, Chalmers L, Bereznicki LR, Peterson GM, Bimirew MA, Kassie DM. Barriers and facilitators of adherence to antiretroviral drug therapy and retention in care among adult HIV-positive Patients: A qualitative study from Ethiopia. PLoS One. 2014;9:e97353 Public Library of Science.

40. Azia IN, Mukumbang FC, van Wyk B. Barriers to adherence to antiretroviral treatment in a regional hospital in Vredenburg, Western Cape, South Africa. South Afr J HIV Med. 2016;17 [cited 2020 Aug 31]. Available from: https:// www.ncbi.nlm.nih.gov/pmc/articles/PMC5843173/.

41. Sereti I, Sheikh V, Shaffer D, Phanuphak N, Gabriel E, Wang J, et al. Prospective international study of incidence and predictors of immune reconstitution inflammatory syndrome and death in people living with human immunodeficiency virus and severe lymphopenia. Clin Infect Dis. 2020;71:652-60 Oxford Academic.

42. Novak RM, Richardson JT, Buchacz K, Chmiel JS, Durham MD, Palella FJ, et al. Immune reconstitution inflammatory syndrome: incidence and implications for mortality. AIDS. 2012;26:721-30.
43. UNAIDS. Fast-Track - Ending the AIDS epidemic by 2030 [Internet]. 2014 [cited 2020 Jun 6]. Available from: https://www.unaids.org/en/resources/ documents/2014/JC2686_WAD2014report.

44. Assefa Y, Hill PS, Van Damme W, Dean J, Gilks CF. Leaving no one behind: lessons from implementation of policies for universal HIV treatment to universal health coverage. Glob Health. 2020;16:17.

45. Geng EH, Havlir DV. The science of rapid start-from the when to the how of antiretroviral initiation. PLoS Med. 2017;14:e1002358.

46. Penn AW, Azman H, Horvath H, Taylor KD, Hickey MD, Rajan J, et al. Supportive interventions to improve retention on ART in people with HIV in low- and middle-income countries: A systematic review. PLoS One. 2018;13: e0208814 Public Library of Science.

47. Fox MP, Pascoe S, Huber AN, Murphy J, Phokojoe M, Gorgens M, et al. Adherence clubs and decentralized medication delivery to support patient retention and sustained viral suppression in care: results from a clusterrandomized evaluation of differentiated ART delivery models in South Africa. PLoS Med. 2019:16:e1002874.

48. Ghate M, Zirpe S, Gurav N, Paranjape R, Rewari B, Gangakhedkar R. Transfer out patients receiving antiretroviral therapy from programme clinic: a potential "leak" in the HIV treatment cascade. Sci Res Publishing. 2014;4: 382-6.

49. Wubshet $M$, Berhane $Y$, Worku A, Kebede Y. Death and seeking alternative therapy largely accounted for lost to follow-up of patients on ART in Northwest Ethiopia: A community tracking survey. PLoS One. 2013;8:e59197 Public Library of Science.

50. Chammartin F, Zürcher K, Keiser O, Weigel R, Chu K, Kiragga AN, et al. Outcomes of patients lost to follow-up in African antiretroviral therapy programs: individual patient data meta-analysis. Clin Infect Dis. 2018;67: $1643-52$.

\section{Publisher's Note}

Springer Nature remains neutral with regard to jurisdictional claims in published maps and institutional affiliations.

Ready to submit your research? Choose BMC and benefit from:

- fast, convenient online submission

- thorough peer review by experienced researchers in your field

- rapid publication on acceptance

- support for research data, including large and complex data types

- gold Open Access which fosters wider collaboration and increased citations

- maximum visibility for your research: over $100 \mathrm{M}$ website views per year

At BMC, research is always in progress.

Learn more biomedcentral.com/submissions 